\title{
Complex Total Hip Arthroplasty in a Young Patient with Previous Acetabulum Fracture and Exposed Intra-Articular Hardware: A Case Report and Review of the Literature
}

\author{
Kyle Andrews M.D., Lucas McKean B.S., Vithal Shendge M.D. \\ Department of Orthopaedic Surgery, University of Toledo Medical Center, Toledo, OH, US. \\ Kyle.Andrews3@UToledo.edu
}

\begin{abstract}
Acetabular fractures can complicate hip arthroplasty and result in higher rates of intraoperative complications and worse long-term outcomes ${ }^{1}$. Previous studies have shown increased rates of acetabular-sided complications and worse long-term outcomes in the setting of prior acetabular fractures. We present a total hip arthroplasty complicated by severe post-traumatic arthritis and a vascular necrosis. The patient was found to have a Paprosky Type IIA acetabular defect with exposed intra-articular hardware due to aprevious both-column acetabulum fracture treated with open reduction and internal fixation. Cancellous autograft was used for acetabular reconstruction and a cementless acetabular cup was inserted, as survival has been shown to be superior to cemented cups in patients with acetabular deficiency. Subsequent follow-up showed well fixated components with a good functional outcome in this patient.
\end{abstract}

\section{INTRODUCTION}

Total hip arthroplasty (THA) has drastically improved the quality of life in patients dealing with debilitating conditions of the hip, and has even been called the operation of the century ${ }^{2}$. With the advance of surgical techniques, we are performing total hip arthroplasty in more complicated patients including patients with complex acetabular pathology. However, studies have shown increased complication and revision rates in patients with a history of acetabulum fractures when compared with patients undergoing THA for primary osteoarthritis or avascular necrosis $(\mathrm{AVN})^{1}$. Overall intermediate survivorship has been shown to be lower in these patients due to aseptic loosening, infection, dislocation, and heterotopic ossification. It has been reported that THA survivorship is independent of fracture management: open reduction and internal fixation (ORIF) versus non-operative treatment ${ }^{1}$. Despite the challenges, Roth et. al. reported positive outcomes in $87 \%$ of patients ten years post-operatively who underwent THA with a history of previous acetabular fracture ${ }^{3}$.

We now report a complex primary THA in a young patient with a previous both-column acetabular fracture status-post ORIF oneyear prior who went on to develop severe post-traumatic arthritis and AVN with subsequent limb-length discrepancy and acetabular bone loss with exposed intra-articular hardware. A posterolateral approach was utilized. Acetabular bone grafting was performed using healthy femoral neck cancellous autograft. A similar case report noted a positive outcome three years post-operatively in a patient who underwent acetabular ORIF followed by autograft acetabular reconstruction and cementless THA ${ }^{4}$.

\section{Case Presentation}

This patient is a 46 year old female referred to our clinic for right hip pain. She was riding a bicycle one year prior when she was struck by a vehicle. She went to an outside facility with multiple injuries including a right 
Complex Total Hip Arthroplasty in a Young Patient with Previous Acetabulum Fracture and Exposed Intra-Articular Hardware: A Case Report and Review of the Literature

both column acetabulum fracture and left sacroiliac (SI) joint disruption. She also had a right tibia fracture, multiple fractured ribs, and various abdominal injuries. The both-column acetabular fracture was treated with open reduction and internal fixation (ORIF) through a Stoppa approach with a separate lateral window at the outside facility. Percutaneous screws were placed across the left SI joint. Her post operative course was complicated by a bladder injury requiring reopening of the Stoppa incision, bladder repair, and a suprapubic catheter. She has gone on to heal all her wounds but complained of persistent and severe, aching right groin pain for one-year duration. She has been unable to bear weight on her right lower extremity over the last year. She is a one-half pack-per-day smoker on chronic narcotics. In addition, she has low back pain with radicular symptoms likely secondary to scoliotic deformity and pelvic obliquity due to shortening of the right lower extremity of approximately five centimeters. Clinically, the patient showed evidence of lower extremity wasting due to disuse. Right hip aspiration was negative for infection two months prior. X-rays (Figure 1) and CT scans reveal severe post-traumatic arthritis and AVN of the right hip with resultant superomedial migration of the acetabulum and limb-length discrepancy. Hardware was observed anterior, posterior, and superior to the acetabulum with possibility of at least threeintra-articular screws.

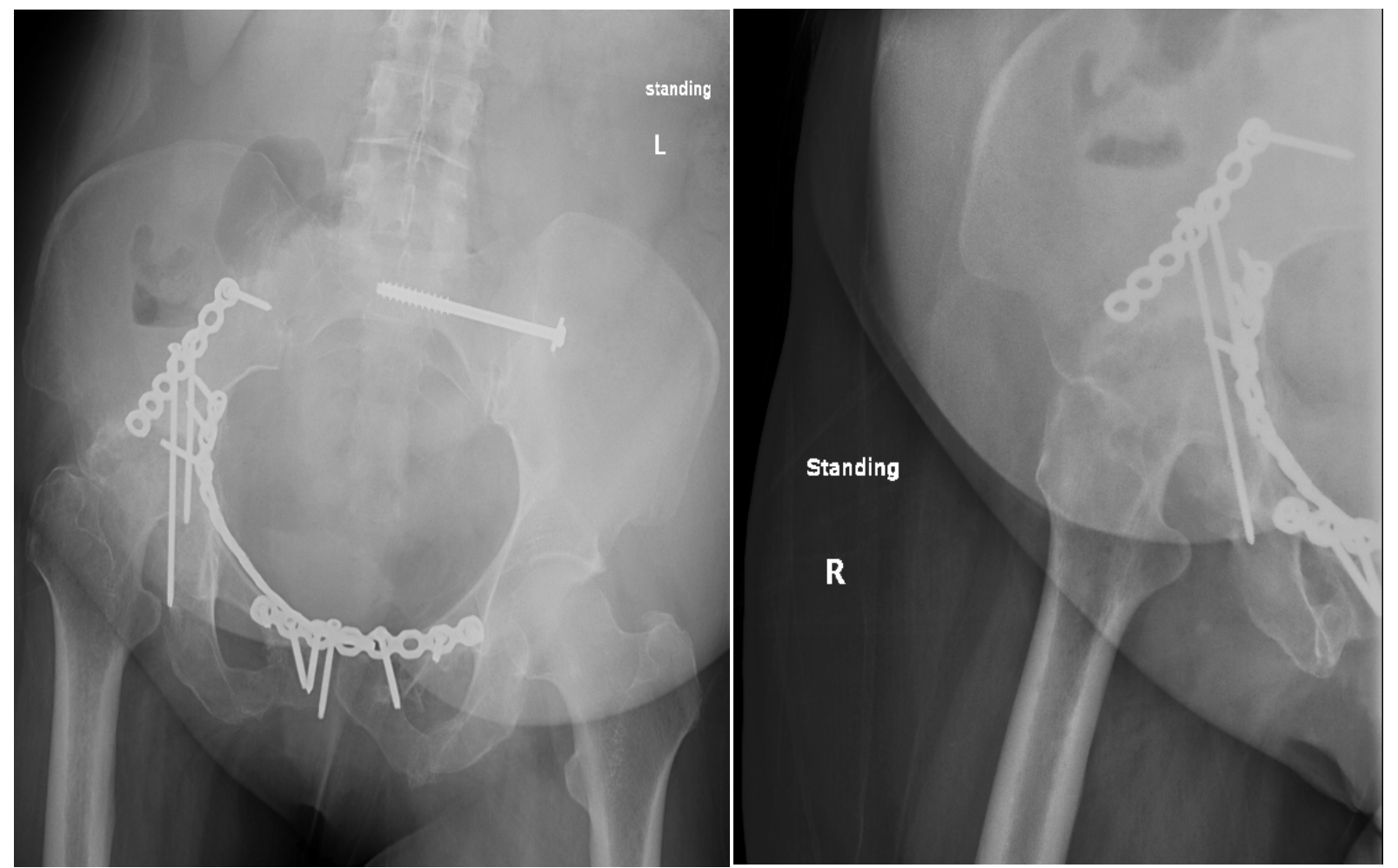

Fig1. Plain films demonstrating previous acetabular and sacro-iliac joint fixation with acetabular bone loss and migration secondary to post-traumatic arthritis and avascular necrosis.

Total hip arthroplasty was planned with anticipation of acetabular bone graft augmentation and possible diamond tipped burr for reaming of intra-articular hardware. The risks, benefits, and alternatives of the procedure were explained and the patient elected to proceed. The patient was brought to the operating room and positioned in the right lateral decubitus position and a standard posterior approach to the hip was utilized. Once dissection was carried down to the hip joint and femoral neck cut made, the femoral head was extracted and observed to be completely atrophic (Figure 2). 
Complex Total Hip Arthroplasty in a Young Patient with Previous Acetabulum Fracture and Exposed Intra-Articular Hardware: A Case Report and Review of the Literature
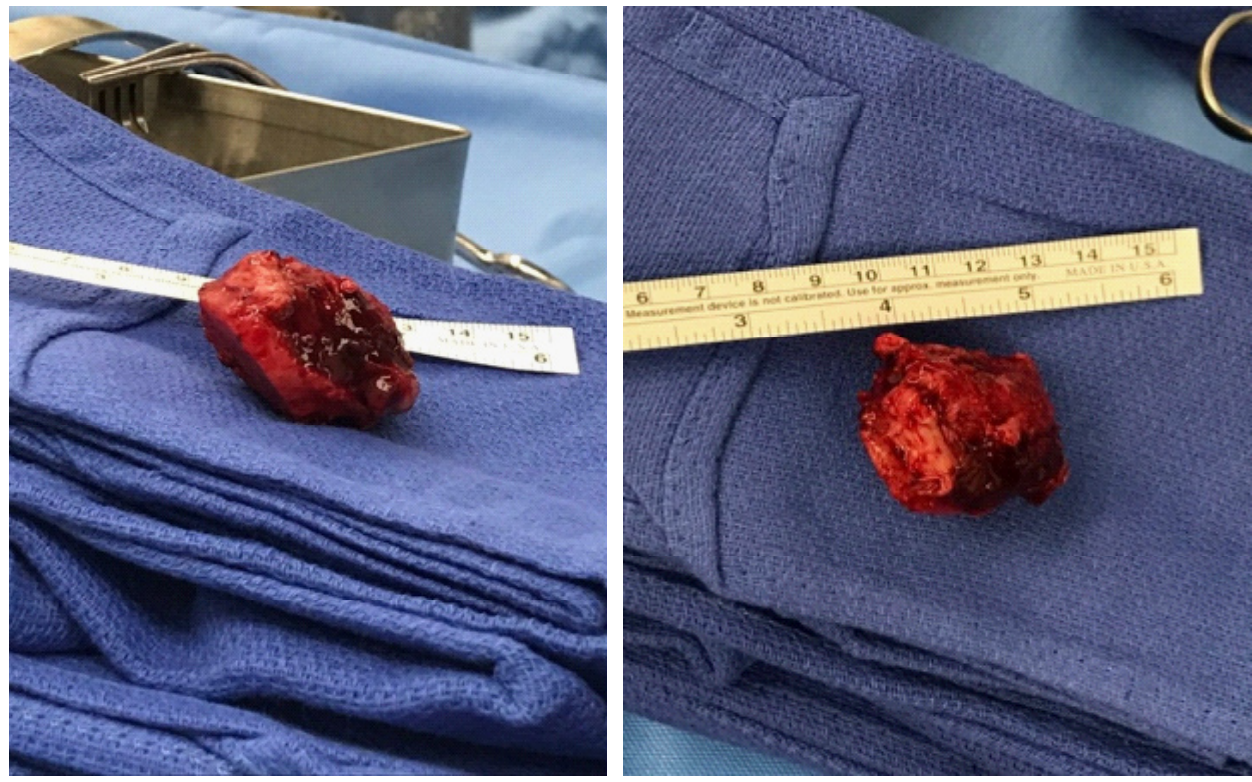

Fig2. Intraoperative pictures demonstrating the atrophic femoral head.

The neck was found to be relatively healthy and therefore the cancellous bone was harvested for acetabular defect reconstruction. Following excision of the acetabular scar tissue and serial reaming, the acetabular rim was intact, but there was superomedial bone loss resulting in visualization of the anterior column plate and associated screws (Figure 3).

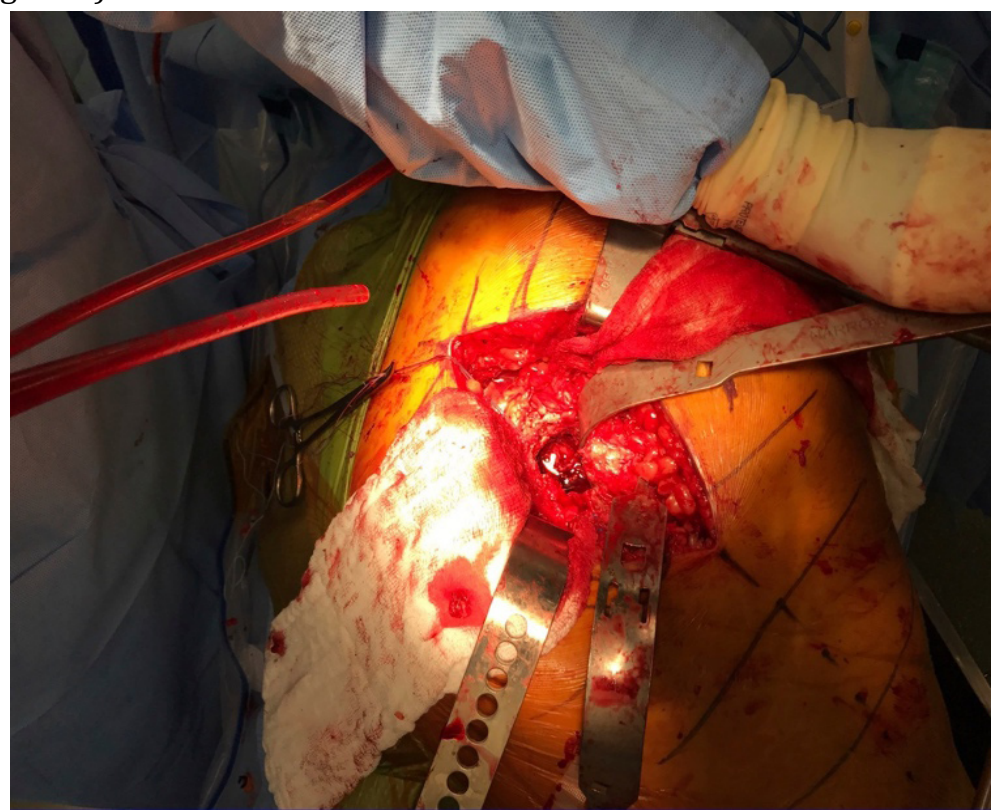

Fig3. Intraoperative photograph of the medial acetabular wall defect and exposed anterior column plate.

The femoral neck cancellous autograft was then used to reconstruct the acetabular defect. A titanium hemispherical cluster hole shell was impacted into position and secured with two cancellous screws. Amodular dual mobility polyethylene liner was then inserted. The femur was then broached and an appropriately sized femoral stem was malleted into position. Due to the patient's young age, a ceramic femoral head was chosen. 
Upon reduction, the hip was found to be stable with adequate offset and appropriate limb-length correction. A capsular repair was performed followed by wound closure in standard fashion. Post-operative plain films demonstrated a well-fixed right total hip arthroplasty with without complication. The patient was admitted to the hospital and allowed to weight-bear as tolerated with posterior hip precautions. Post-operative course was unremarkable and she discharged to home on the second post-operative day.

She has been followed in clinic and at most recent follow-up, eight months after surgery, she is pain free, ambulating with only a small shoe lift, and participating in all activities of daily living. Plain films demonstrate a well-fixed right total hip arthroplasty without complication (Figure 4)

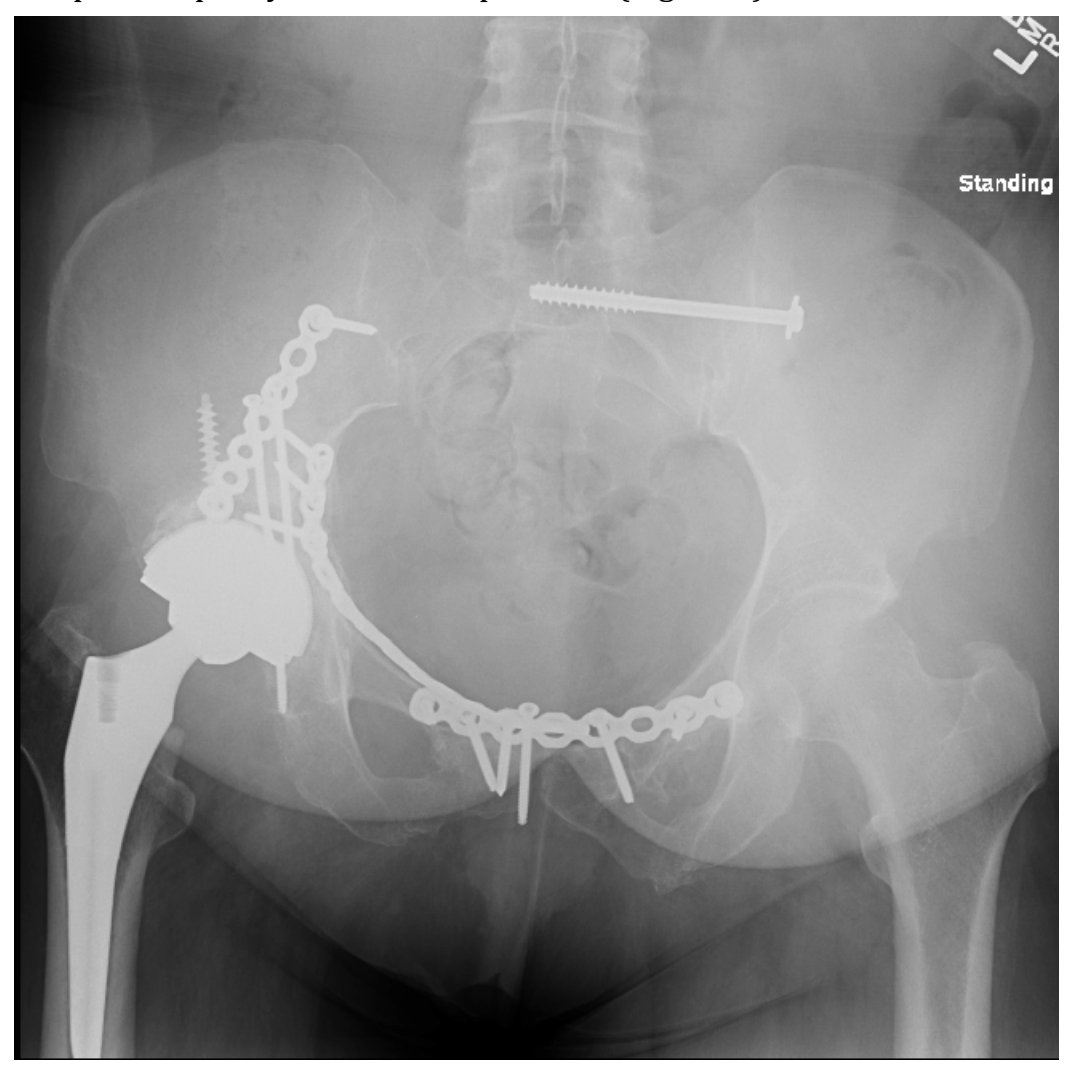

Fig4. Radiograph at eight months post-op.

\section{DISCUSSION}

Previous acetabular fractures complicate the treatment of hip arthritis, and result in higher complication rates in patients that elect THA. Our case was further complicated by exposed hardware within the joint limb-length discrepancy, and superomedial acetabular bone loss (Paprosky Type IIA). A thorough understanding of challenges likely to be encountered is very important towards achieving a successful outcome in these types of cases.

While our patient met several criteria for consideration of THA with Modular Dual Mobility (MDM) implant (neuromuscular disease, prior hip surgery), complicating our decision was the young age of our patient and significant acetabular bone loss with exposed intra-articular hardware. Weber et.al. reported severe bone loss was associated with significantly worse outcomes in THA when compared to outcomes in patients with moderate to mild or no bone loss 5 . It is well established that the extent of acetabular deficiency following ORIF is an important factor in the eventual survival of the acetabular cup in THA. With regard to THA in younger 
Complex Total Hip Arthroplasty in a Young Patient with Previous Acetabulum Fracture and Exposed Intra-Articular Hardware: A Case Report and Review of the Literature

patients, Assiet.al. reported no incidents of dislocation or acetabular-sided failure in 75 patients with an average age of 52.9 years who underwent THA with dual-mobility cups followed for an average of nearly 6 years. ${ }^{12}$ Additionally, Puch et.al. reported no difference in long-term outcomes in patients grouped by age (average 49.9 years versus 72.3 years) who underwent THA with dual-mobility cup. Average follow-up was nearly 14 years with reported acetabular survival $98.4 \% .{ }^{11}$ These studies suggest total hip arthroplasty may be a viable option in younger patients with post-traumatic arthritis when using newer cementless, modular implants. With newer implants showing improved survival in younger patients, earlier intervention with THA may improve outcomes compared to delayed THA where further bone loss is allowed to progress.

Cementless acetabular fixation is the more popular technique for cup fixation in THA. In this case, superomedial acetabular bone loss complicated the procedure and thus bone grafting was performed to enhance bony in growth into a highly porous acetabular cup. Romnes set.al. found that bone grafting of the acetabulum did not result in inferior outcomes for cementless implants ${ }^{6}$. Additionally, in patients with previously fixed acetabular fractures, Weber et.al. reported zero out of 22 cases of acetabular-sided loosening in patients with a cementless components compared to 15 out of 44 cases of acetabular component loosening in patients with cemented cup fixation ${ }^{5}$. In a separate study, bone grafting of the acetabular defect with implantation of a cementless cup resulted in higher 10 and 20-year survival rates than bone grafting with cemented cups ${ }^{8}$. These results suggest that patients undergoing THA with acetabular bone loss would benefit from acetabular reconstruction followed by cementless implants as opposed to cemented acetabular components.

\section{CONCLUSION}

In this case report we discuss the complexities of total hip arthroplasty in a patient with a prior acetabular fracture. The procedure was complicated by intra-articular hardware, limb-length discrepancy, and superomedial acetabular bone loss (Paprosky Type IIA) requiring bone graft reconstruction. Despite the challenges, a well-fixed modular dual mobility cup was placed without complication. The femoral component was also press-fit into place and a ceramic head was chosen due to the patient's young age. At eight month follow-up, plain films show a well-fixed total hip arthroplasty with no complications. The patient has an improved limb-length discrepancy and is participating in all desired activities. Acetabular bone stock is an important factor for THA success and incidence of acetabular deficiency in young patients is on the rise. However, there is evidence to suggest early arthroplasty may result in better long-term outcomes as bone loss is minimized.

\section{REFERENCES}

1. Morison, Z., Moojen, D. J., Nauth, A., Hall, J., Mckee, M. D., Waddell, J. P., \&Schemitsch, E. H. (2015). Total Hip Arthroplasty After Acetabular Fracture Is Associated With Lower Survivorship and More Complications. Clinical Orthopaedics and Related Research ${ }^{\circledR}, 474(2), 392-398$. doi:10.1007/ s11999-015-4509-1

2. Learmonth, I. D., Young, C., \&Rorabeck, C. (2007). The operation of the century: total hip replacement. The Lancet, 370(9597), 1508-1519. doi:10.1016/s0140-6736(07)60457-7

3. Roth, P. V., Abdel, M. P., Harmsen, W. S., \& Berry, D. J. (2015). Total Hip Arthroplasty After Operatively Treated Acetabular Fracture. The Journal of Bone and Joint Surgery-American Volume, 97(4), 288-291. doi:10.2106/ jbjs.n.00871

4. Gavaskar, A. \& Tummala, N. (2012). Delayed cementless total hip arthroplasty for neglected dislocation of hip combined with complex acetabular fracture and deficient bone stock. Chinese Journal of Traumatology, 15(6), 370-372. Retrieved March 10, 2017, from https://www.ncbi.nlm.nih.gov/pubmed/23186930.

American Research Journal of Orthopedics and Traumatology

Page 5 
Complex Total Hip Arthroplasty in a Young Patient with Previous Acetabulum Fracture and Exposed Intra-Articular Hardware: A Case Report and Review of the Literature

5. Weber, M., Berry, D. J., \& Harmsen, W. S. (1998). Total Hip Arthroplasty after Operative Treatment of an Acetabular Fracture*. The Journal of Bone \& Joint Surgery, 80(9), 1295-1305. doi:10.2106/00004623-199809000-00008

6. Romness, D. M., \& Lewallen, D. G. (1990). Total hip arthroplasty after fracture of the acetabulum. Long-term results. The Bone and Joint Journal, 72-B(5), 761-764. Retrieved March 13, 2017, from http://www.bjj. boneandjoint.org.uk/content/jbjsbr/72-B/5/761.full.pdf

7. Angadi, D. S., Brown, S., \& Crawfurd, E. J. (2012). Cemented polyethylene and cementless porous-coated acetabular components have similar outcomes at a mean of seven years after total hip replacement: A prospective randomised study. The Bone \& Joint Journal, 94-B(12), 1604-1610. doi:10.1302/0301620x.94b12.28060

8. Sternheim, A., Abolghasemian, M., Safir, O. A., Backstein, D., Gross, A. E., \& Kuzyk, P. R. (2013). A Long-Term Survivorship Comparison Between Cemented and Uncemented Cups With Shelf Grafts in Revision Total Hip Arthroplasty After Dysplasia. The Journal of Arthroplasty, 28(2), 303-308. doi:10.1016/j.arth.2012.06.004

9. Schreurs BW, Busch VJ, Welten ML, Verdonschot N, Sloof TJ, Gardeniers JW. Acetabular reconstruction with impaction bone-grafting and a cemented cup in patients younger than fifty years old. J Bone Joint Surg Am. 2004 Nov; 86-A(11): 2385-92.

10. Busch VJ, Gardeniers JW, Verdonschot N, Slooff TJ, Schreurs BW. Acetabular reconstruction with impaction bone-grafting and a cemented cup in patients younger than fifty years old: a concise follow-up, at twenty to twenty-eight years, of a previous report. J Bone Joint Surg Am. 2011 Feb 16;93(4):367-71.

11. Puch, J., Derhi, G., Descamps, L., Verdier, R., \& Caton, J. H. (2016). Dual-mobility cup in total hip arthroplasty in patients less than fifty five years and over ten years of follow-up. International Orthopaedics, 41(3), 475-480. doi:10.1007/s00264-016-3325-x

12. Assi, C., El-Najjar, E., Samaha, C., \&Yammine, K. (2017). Outcomes of dual mobility cups in a young Middle Eastern population and its influence on life style. International Orthopaedics, 41(3), 619-624. doi:10.1007/ s00264-016-3390-1

Citation: Kyle Andrews M.D., Lucas McKean B.S., Vithal Shendge M.D. “Complex Total Hip Arthroplasty in a Young Patient with Previous Acetabulum Fracture and Exposed Intra-Articular Hardware: A Case Report and Review of the Literature". American Research Journal of Orthopedics and Traumatology. 2017; 2(1): 1-6.

Copyright (c) 2017 Kyle Andrews M.D., Lucas McKean B.S., Vithal Shendge M.D. This is an open access article distributed under the Creative Commons Attribution License, which permits unrestricted use, distribution, and reproduction in any medium, provided the original work is properly cited. 